\title{
Automatic Segmentation of Pulmonary Structures in Chest CT Images
}

\author{
Yeny Yim $^{1}$ and Helen Hong ${ }^{2, *}$ \\ ${ }^{1}$ School of Electrical Engineering and Computer Science, Seoul National University \\ shine@cglab.snu.ac.kr \\ ${ }^{2}$ School of Computer Science and Engineering, BK21: Information Technology, \\ Seoul National University, San 56-1 Shinlim 9-dong Kwanak-gu, Seoul 151-742, Korea \\ hlhong@cse.snu.ac.kr
}

\begin{abstract}
We propose an automatic segmentation method for accurately identifying lung surfaces, airways, and pulmonary vessels in chest CT images. Our method consists of four steps. First, lungs and airways are extracted by inverse seeded region growing and connected component labeling. Second, pulmonary vessels are extracted from the result of first step by gray-level thresholding. Third, trachea and large airways are delineated from the lungs by three-dimensional region growing based on partitioning. Finally, accurate lung regions are obtained by subtracting the result of third step from the result of first step. The proposed method has been applied to 10 patient datasets with lung cancer or pulmonary embolism. Experimental results show that our segmentation method extracts lung surfaces, airways, and pulmonary vessels automatically and accurately.
\end{abstract}

\section{Introduction}

Chest computed tomography (CT) is widely used to evaluate numerous lung diseases, including lung nodules, pulmonary embolism and emphysema [1]. A precursor to all of these applications is the lung segmentation. In particular, since multi-detector row CT scanner routinely generate 300 or more two-dimensional (2D) slices per patient, it is critical to develop an efficient method for automatically segmenting the precise lung boundaries, airways and pulmonary vessels.

Several methods have been suggested for segmentation of lungs in chest CT scans. In Denison [2], manually traced boundaries were used to estimate regional gas and tissue volumes in the lungs of normal subjects. In Hedlund [3], 3D region growing with manually specified seed points was presented for segmenting the lungs. However, these manual and semi-automatic methods are laborious and subject to inter- and intra-observer variations. Brown [4] proposed an automatic, knowledgebased method for segmenting the chest CT images. Anatomic knowledge stored in a semantic network is used to guide the segmentation process. In knowledge-based method, accuracy significantly depends on the level of knowledge. In Armato [5],

* Corresponding author. 
gray-level thresholding is used to segment the thorax from the background and then the lungs from the thorax. A rolling-ball algorithm is applied to the lung segmentation contours to avoid the loss of juxtapleural nodules. This method was for use as a preprocessing step for automated lung nodule detection and mesothelioma measurement. In $\mathrm{Hu}$ [6], gray-level thresholding is used to distinguish between the low density lung regions and denser surrounding tissue. The radiodense pulmonary vessels are excluded from the lung regions through the gray-level thresholding so that holes in the lung surface near the mediastimum are made. To fill these holes, 2D morphological closing is used. However, unsmooth boundaries of lungs are still remained. To solve this problem, Ukil [7] proposed an automatic method for the 3D smoothing of the lung boundaries using 3D morphological closing with an ellipsoidal kernel.

Current approaches still need more progress in computational efficiency and accuracy for segmenting lungs in chest CT scans. In this paper, we describe an automatic segmentation method for accurately identifying pulmonary structures such as lung surfaces, airways, and pulmonary vessels in chest CT images. First, a similar operation to region growing is used to segment the thorax from the background and then the lungs and airways from the thorax. To remove other low-density regions which have similar intensity with the lungs, connected component labeling is used. Second, pulmonary vessels are extracted from the result of first step by gray-level thresholding. Third, trachea and large airways are delineated from the lungs by 3D region growing based on partitioning. Finally, accurate lung regions are obtained by subtracting the result of third step from the result of first step. To evaluate the accuracy, we present results comparing automatically extracted borders by proposed method to manually traced borders from two radiologists. We also compare the results of two automatic segmentation methods: our proposed method and commercial tool Analyze. Experimental results show that our segmentation method extracts pulmonary structures accurately and automatically. Accurate and automatic segmentation would be more useful for clinical applications of pulmonary nodule detection, pulmonary embolism and emphysema analysis.

The organization of the paper is as follows. In Section 2, we discuss how to extract the pulmonary structures from other organs in chest CT images. In Section 3, experimental results show how the method accurately and automatically segments the pulmonary structures in the chest CT images. This paper is concluded with a brief discussion of the results in Section 4.

\section{Segmentation of Pulmonary Structures}

For the segmentation of the chest CT images, we apply the pipeline shown in Fig. 1. Since our method is applied to the pulmonary nodule matching and pulmonary embolism analysis, we assume that each CT scan is acquired at the maximal inspiration and the dataset includes the thorax from the trachea to the diaphragm. 


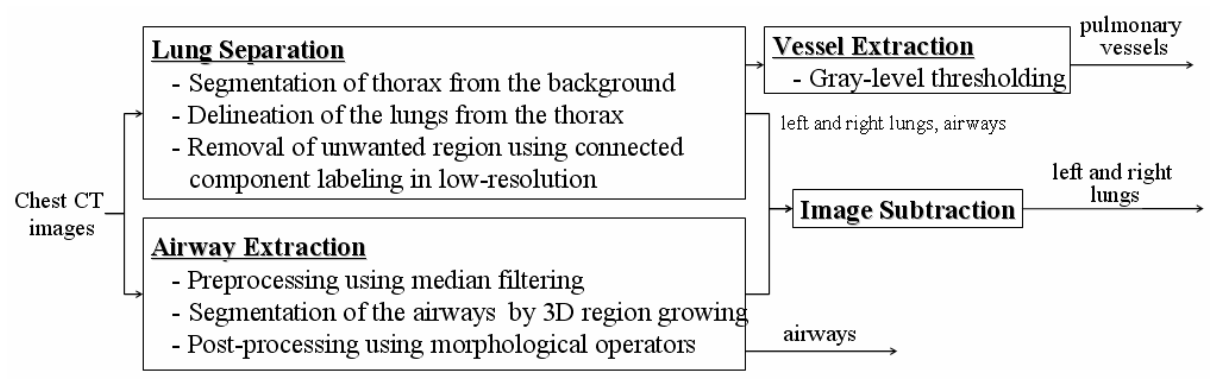

Fig. 1. The pipeline of the automatic lung segmentation

\subsection{Threshold Selection Using Optimal Thresholding}

For this step, we assume that the image volume contains only two principal brightness regions: 1) high-density regions within the chest wall structures, 2) low-density regions in the lungs. We use optimal thresholding [8] to automatically select a threshold for separating thorax from the lung regions. The segmentation threshold is selected through iterative procedure. We first select the initial threshold T0 and apply T0 to the volume to separate the voxels into high-density and low-density regions. The new threshold for next step is the average of the mean gray-levels of two regions. This threshold update procedure is repeated until there is no change in the threshold. Since the tissue density of CT images varies between subjects according to radiation dose of CT scanner, optimal thresholding allows us to adapt these variations.

\subsection{Lung Separation Using 2D Inverse Seeded Region Growing}

The goal of this step is to separate voxels of lung tissue from the surrounding anatomy. Generally, thresholding and 3D region growing are used to identify lungs [6]. Since these methods based on difference in attenuation values can produce holes in high-density vessels within the lungs, these holes should be filled by morphological operations such as dilation and erosion [8]. To eliminate the holes, the mask size of these operations has to be larger than the size of holes. However, determining the mask size is difficult to eliminate the holes while distorting the lung region boundaries as little as possible. We propose the $2 \mathrm{D}$ inverse seeded region growing (iSRG) method for the automatic lung separation without these limitations in the chest CT images.

The 2D iSRG is used to automatically segment the thorax from the background and then the lung regions from the thorax, as shown in Fig. 2(b) and (c). In first 2D iSRG, the seed pixel is selected at $(0,0)$ on each $2 \mathrm{D}$ slice, which has a gray level smaller than threshold value selected by optimal thresholding. Background air which surrounds the body is extracted by region growing and then thorax is segmented by inverse operation. In second 2D iSRG, the seed pixel is chosen at a pixel of thorax with a gray level larger than the threshold value. As a result of region growing, thorax region which has similar gray level to the seed pixel is extracted. By inverting the result, we can segment the lungs and airways without inner holes and the distortion of 


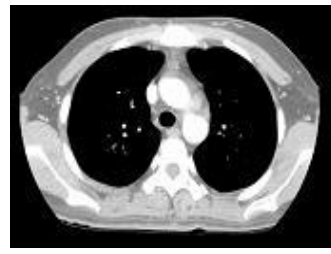

(a)

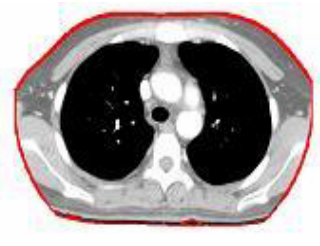

(b)

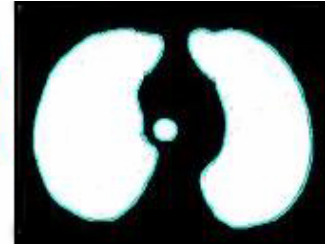

(c)

Fig. 2. The result of lung separation (a) chest CT image (b) thorax extraction from the surrounding anatomy (c) lung delineation from the thorax

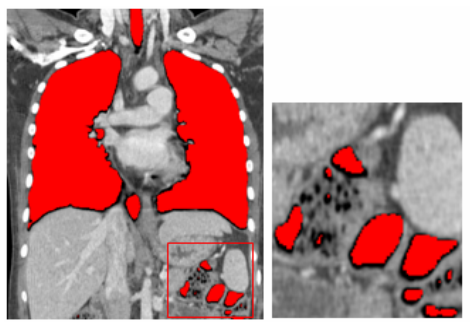

(a)
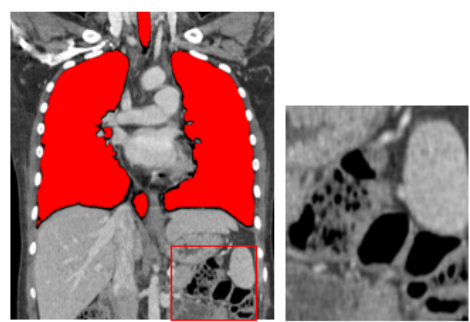

(b)

Fig. 3. The removal of unwanted region (a) the result of $2 \mathrm{D}$ iSRG (b) connected component labeling eliminates bowel gas (indicated by square and displayed by enlarged image)

lung boundaries. Binary images are then constructed as shown in Fig. 2(c). The 3D connected component labeling is applied to ensure that non-pulmonary structures, such as bowel gas, are not erroneously identified as lung regions, as shown in Fig. 3.

After the lung separation, pulmonary vessels are extracted from the above result using gray-level thresholding. All pixels with a gray level larger than the threshold value selected by optimal thresholding are identified as pulmonary vessels.

\subsection{Airway Extraction Using 3D Region Growing Based on Partitioning}

Since the intensities of the trachea and large airways are similar to those of the lungs, the lungs resulting from the lung separation step still contain the trachea and large airways. Thus the airway extraction step segments trachea and large airways by 3D region growing based on partitioning and subtracts the results from the results of lung separation step.

The airway extraction is composed of the following four stages. First, we apply pre-filtering in order to increase robustness of 3D region growing. Due to junctions between the lungs and the airways, the 3D region growing for airway extraction may create an explosion into the lung parenchyma, as shown in Fig. 4(a) and (b). Applying 2D median filter to each slice can make thin these junctions with weak contrast and separate the lungs and the airways, as shown in Fig. 4(c). The mask size for the median filtering is $3 \times 3$. Second, threshold value is selected by applying adaptive 
thresholding. Airway extraction cannot be successful using a single threshold since there are gray-level variations between trachea and large airways. We partition the chest CT images into two parts on the basis of the branching point of trachea. For upper part, a threshold value that is $50 \%$ of the difference between the maximum and minimum values in the image is used. For lower part, predefined threshold value is used. Third, 3D region growing with 26-connectivity is applied to the filtered images. The seed point is automatically selected by searching for the large, circular, air-filled region near the center of the first few slices in the dataset. The regions with a gray level smaller than the threshold value are extracted as the trachea and large airways, as shown in Fig. 4(d). Finally, 2D morphological operators are applied to the results of previous step in which high-density airway wall is not included and unwanted cavities are remained. To prevent these occurrences, we apply a $2 \mathrm{D}$ binary dilation and closing with a $3 \times 3$ mask to each slice repeatedly. We partition the chest $\mathrm{CT}$ images into two parts and apply different number of iteration to each part.

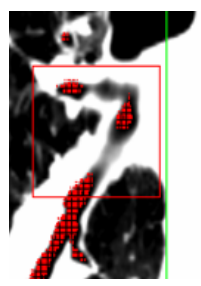

(a)

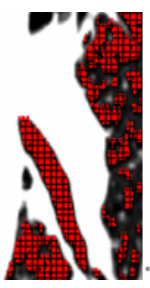

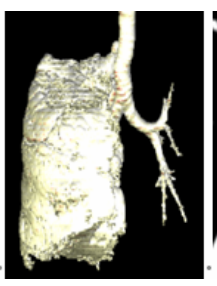

(b)

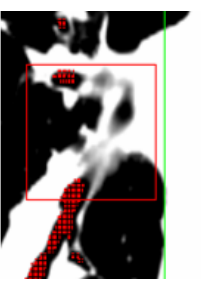

(c)
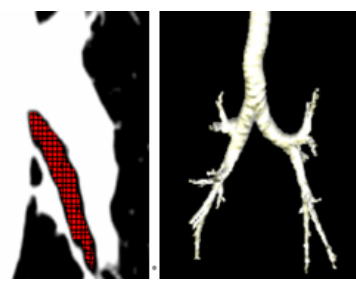

(d)

Fig. 4. The effect of median filtering (a)(b) the result of airway extraction without median filtering $(c)(d)$ the result of airway extraction with median filtering

\subsection{Lung Extraction Using Image Subtraction}

After the trachea and large airways are extracted, the results are subtracted from the results of the lung separation. Subtracted images contain only the lung regions. Fig. 5 shows the results of lung extraction by image subtraction. Fig. 5(a) and (b) is the results of lung separation and airway extraction, respectively. Fig. 5(c) is obtained by subtracting Fig. 5(b) from Fig. 5(a).

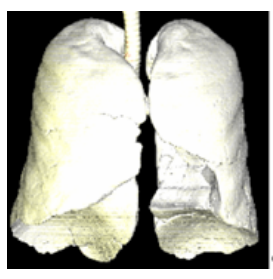

(a)

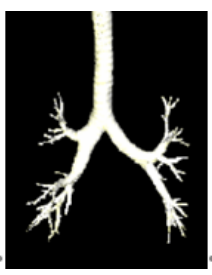

(b)

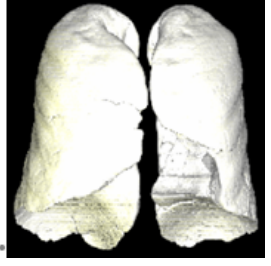

(c)

Fig. 5. The result of lung extraction (a) the result of lung separation (b) the result of airway extraction (c) lungs extracted by image subtraction 


\section{Experimental Results}

All our implementation and test were performed on an Intel Pentium IV PC containing $2.5 \mathrm{GHz}$ and $2.0 \mathrm{~GB}$ of main memory. Our segmentation method has been applied to ten patients with pulmonary nodule or embolism of 16-channel chest CT scans whose properties are described in Table 1. The CT images were obtained with a Philips MX8000 multidectector helical CT scanner or Siemens Sensation16 multidectector helical CT scanner. The image size of all patient datasets is 512 x 512 . The performance of our method is evaluated with the aspects of visual inspection and accuracy and processing time.

Table 1. Image conditions of experimental datasets

\begin{tabular}{|c|c|c|c|c||c|c|c|c|c|}
\hline Subject & Slice \# & $\begin{array}{c}\text { Pixel size } \\
(\mathrm{mm})\end{array}$ & $\begin{array}{c}\text { Slice } \\
\text { thickness } \\
(\mathrm{mm})\end{array}$ & Disease & Subject & Slice \# & $\begin{array}{c}\text { Pixel size } \\
(\mathrm{mm})\end{array}$ & $\begin{array}{c}\text { Slice } \\
\text { thickness } \\
(\mathrm{mm})\end{array}$ & Disease \\
\hline \hline 1 & 258 & $0.6 \times 0.6$ & 1.5 & PE & 6 & 358 & $0.64 \times 0.64$ & 2.0 & PN \\
\hline 2 & 209 & $0.77 \times 0.77$ & 1.5 & PE & 7 & 270 & $0.57 \times 0.57$ & 2.0 & PN \\
\hline 3 & 456 & $0.61 \times 0.61$ & 0.75 & PE & 8 & 371 & $0.6 \times 0.6$ & 2.0 & PN \\
\hline 4 & 372 & $0.68 \times 0.68$ & 0.75 & PE & 9 & 407 & $0.62 \times 0.62$ & 2.0 & PN \\
\hline 5 & 374 & $0.61 \times 0.61$ & 0.75 & PE & 10 & 446 & $0.55 \times 0.55$ & 2.0 & PN \\
\hline
\end{tabular}

(PE: pulmonary embolism, PN: pulmonary nodule)

Fig. 6 shows the results of lung segmentation of subject 1, 6, 7. The first row shows the $2 \mathrm{D}$ binary image and the second row shows the $3 \mathrm{D}$ display of segmented lungs. These results show our proposed method segments lung boundaries with high curvature precisely.

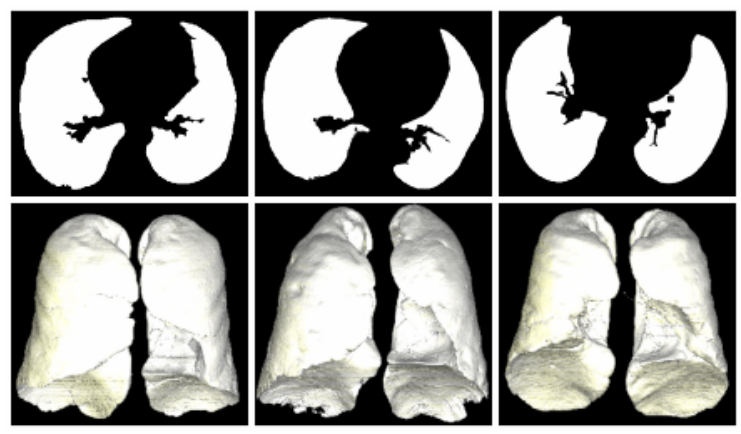

Fig. 6. The results of automatic lung segmentation

Fig. 7 shows the results of airway extraction of subject 1, 3, 6 and pulmonary vessel extraction of subject $3,6,8$. These results show that our proposed method extracts the airways and pulmonary vessels accurately. 

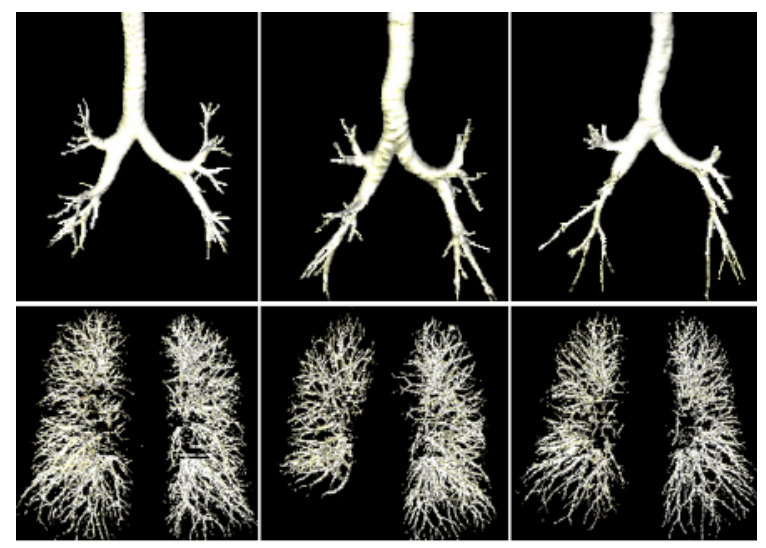

Fig. 7. The results of airway extraction and vessel extraction

To evaluate the accuracy of lung segmentation, we performed two comparisons. First, we compared our method with manual method. Second, considering the manual method as gold standard, we compared our method with commercial tool Analyze (Mayo clinic, Rochester, USA). For manual method, two radiologists manually outlined the left and right lung borders for 10 patient datasets. For the first comparison, the accuracy was measured by computing the mean, rms, and maximum distance between computer-defined contour and the manually-outlined contour. For each pixel on the computer-defined contour, the minimum distance to the manuallyoutlined contour was computed as

$$
d_{i}=\min _{j}\left\|X_{i}^{C}-X_{j}^{M}\right\|
$$

where $\mathrm{X}_{\mathrm{i}}{ }^{\mathrm{C}}$ is the computer-defined contour pixel location and $\mathrm{X}_{\mathrm{j}}{ }^{\mathrm{M}}$ is the manuallyoutlined contour pixel location.

Fig. 8 shows a comparison between the computer-defined contours and the manually-outlined contours. The figure shows the difference between radiologist 1 and

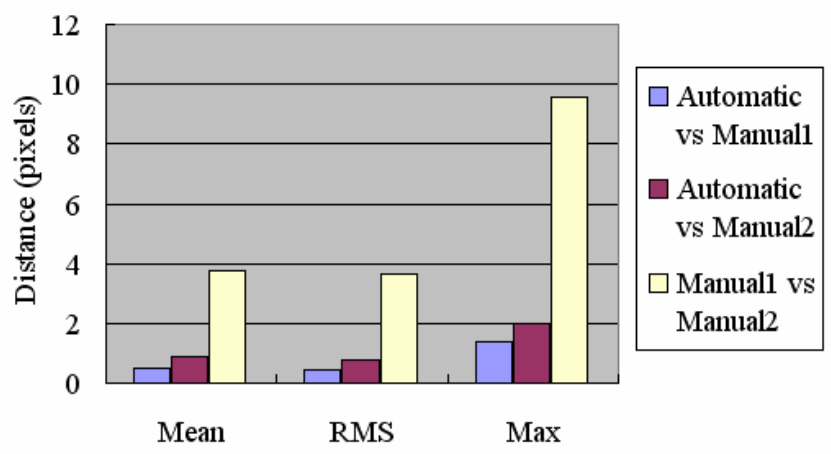

Fig. 8. The accuracy evaluation using distance measure 
the computer, and the difference between radiologist 2 and the computer. In addition, inter-observer variations are evaluated by computing the distances between the manually-outlined contours. The difference between the radiologists and the computer could be considered not significant since the variations between the computer and any of the radiologists is smaller in magnitude than the variations between two radiologists.

For the second comparison, considering the manual method as gold standard, the accuracy was measured by computing the number of error voxels and error rates of two automatic methods: our method and Analyze. Table 2 shows the average number of error voxels and the error rates of proposed method is much smaller than those of Analyze.

Table 2. Average number of error pixels and error rates

\begin{tabular}{|c|c|c|}
\hline $\begin{array}{c}\text { Average number of } \\
\text { error voxels }\end{array}$ & Analyze & Proposed method \\
\hline Error rates & $1,607,006$ & 336,975 \\
\hline
\end{tabular}

Total processing time is summarized in Table 3 where execution time is measured for lung separation and vessel extraction, airway and lung extraction processes. On average, 22.7 seconds are required to segment $512 \times 512 \times 352$ dataset.

Table 3. Total processing time (sec)

\begin{tabular}{|c|c|c|c||c|c|c|c|}
\hline Subject & A & B & $\begin{array}{c}\text { Total Processing } \\
\text { Time }\end{array}$ & Subject & A & B & $\begin{array}{c}\text { Total Processing } \\
\text { Time }\end{array}$ \\
\hline \hline 1 & 14.704 & 2.515 & 17.219 & 6 & 20.391 & 4.265 & 24.656 \\
\hline 2 & 7.625 & 1.922 & 9.547 & 7 & 14.188 & 2.719 & 16.907 \\
\hline 3 & 24.063 & 5.468 & 29.531 & 8 & 19.329 & 3.468 & 22.797 \\
\hline 4 & 19.719 & 4.359 & 24.078 & 9 & 23.047 & 4.141 & 27.188 \\
\hline 5 & 20.125 & 4.937 & 25.062 & 10 & 26.578 & 3.937 & 30.515 \\
\hline
\end{tabular}

(A : Lung Separation and Vessel Extraction, B : Airway and Lung Extraction)

\section{Conclusion}

We have developed an automatic method for accurately identifying pulmonary structures in the chest CT images. Our automatic segmentation extracts accurate lung surfaces, airways and pulmonary vessels. In first step, using 2D iSRG and connected component labeling, the airways and the lungs can be accurately extracted without hole-filling. In particular, connected component labeling in low-resolution can reduce the memory use and computation time. Pulmonary vessels can be identified from the result of first step by gray-level thresholding. In second step, trachea and large airways can be accurately delineated from the lungs by splitting the chest CT image into two parts and applying different threshold values. Accurate lung regions can be 
identified by subtracting the trachea and large airways from the airways and the lungs. Ten patient datasets with lung cancer or pulmonary embolism have been used for the performance evaluation with the aspects of visual inspection and accuracy and processing time. The results of our method show that lungs with large curvature, airways and pulmonary vessels are accurately extracted. The comparison with manual analysis shows that the root mean square difference between the computer and manual analysis is about 0.8 pixels. The difference could be considered not significant since the variations between the computer and any of the radiologists is smaller in magnitude than inter-observer variations. The comparison with Analyze shows that error rates of our method is $13 \%$ smaller than those of Analyze for 10 patient datasets. On average, 22.7 seconds are required to segment 512 × 512 × 352 dataset. Proposed method can be successfully used for lung nodule matching and CT lung perfusion, which are preprocessing step for pulmonary nodule detection and pulmonary embolism analysis, respectively.

\section{Acknowledgements}

This work was supported in part by a grant B020211 from Strategic National R\&D Program of Ministry of Science and Technology and a grant 10014138 from the Advanced Technology Center Program and the Brain Korea 21 Project. The ICT at Seoul National University provides research facilities for this study.

\section{References}

1. Remy-Jardin M., Remy J., Spiral CT of the Chest, Berlin: Springer-Verlag (1996).

2. Denison, D. M., Morgan, M. D. L., Millar, A. B., Estimation of regional gas and tissue volumes of the lung in supine man using computed tomography, Thorax, Vol. 41 (1986) 620-628.

3. Hudlend, L.W., Anderson, R.F., Goulding, P.L., Beck, J.W., Effmann, E.L., Putman, C.E., Two methods for isolating the lung area of a CT scan for density information, Radiology, Vol. 144 (1982) 353-357.

4. Brown, M. S., McNitt-Gray, M. F., Mankovich, N. J., Goldin, J. G., Hiller, J., Wilson, L. S., Aberle, D. R., Method for segmenting chest CT image data using an anatomic model: Preliminary results, IEEE Trans. Medical Imaging Vol. 16, No. 6 (1997) 828-839.

5. ArmatoIII, S.G., Sensakovic, W.F., Automated Lung Segmentation for Thoracic CT: Impact on Computer-Aided Diagnosis, Academic Radiology Vol.11 (2004) 1011-1021.

6. Hu, S., Hoffman, E.A., Reinhardt, J.M., Accurate Lung Segmentation for Accurate Quantitation of Volumetric X-Ray CT Images, IEEE Transactions on Medical Imaging Vol. 20, No. 6 (2001) 490-498.

7. Ukil, S., Reinhardt, J.M., Smoothing Lung Segmentation Surfaces in 3D X-ray CT Images using Anatomic Guidance, In Proc. SPIE Conf. Medical Imaging Vol.5340 (2004) 10661075 .

8. R.G.Gonzalez, R.E.Woods, Digital Image Processing, 1st Ed. (1993). 